
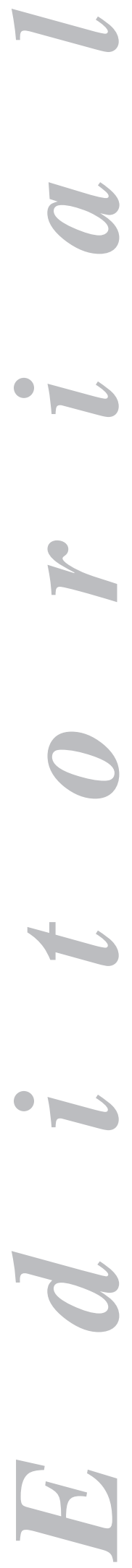

\title{
Calprotectina fecal
}

\section{Características generales}

La calprotectina (CP) es una proteína que se encuentra en el organismo de forma abundante y ampliamente distribuida. Va unida al calcio y pertenece a la familia S100. Está contenida principalmente en los leucocitos polimorfonucleares neutrófilos (PMNs) y también está presente, aunque en una menor proporción, en los monocitos y los macrófagos reactivos (1).

La CP representa aproximadamente el 5\% del contenido total proteico de los leucocitos neutrófilos y un $60 \%$ del contenido total de proteínas que están presentes en su citoplasma (2).

Presenta claras propiedades bacteriostáticas y fungicidas y sus niveles plasmáticos se elevan de 5 a 40 veces, en presencia de procesos infecciosos y/o inflamatorios (3).

También se encuentra presente en las heces y la concentración fecal de calprotectina (CPF) es muy superior comparada con sus niveles plasmáticos (aproximadamente unas 6 veces) (4).

Se encuentran niveles elevados de $\mathrm{CP}$ en las heces de pacientes con diversos procesos inflamatorios intestinales, tanto localizados a nivel del intestino delgado como en cualquier parte del colon (5-7).

\section{Método de determinación de la CPF y valores de referencia}

En 1992 Roseth y cols. (2) desarrollaron el primer método de determinación de $\mathrm{CPF}$, mediante una técnica de ELISA. Desde entonces, se ha mejorado y validado extensamente el método y se emplean muestras muy pequeñas de heces $(0,1 \mathrm{~g})$ que se mezclan con una solución tampón, en un tubo de $5 \mathrm{ml}$ (8). Los resultados de su determinación muestran una muy buena correlación con la excreción fecal en 3 días de granulocitos marcados con un isótopo radiactivo como el Indio 111 (9).

Los nuevos métodos expresan la concentración de CPF en microgramos por gramo, a diferencia de los anteriores que lo medían en miligramos por litro.

La CP es una proteína muy estable y resistente a la degradación proteolítica de las heces. Las muestras pueden ser guardadas hasta 5 días a temperatura normal, sin observar pérdida apreciable de su concentración y se pueden enviar por correo sin refrigeración.

El límite superior de la normalidad se establece en $50 \mu \mathrm{g} / \mathrm{g}$. Los valores de referencia para los niños son similares a los encontrados en los adultos, aunque de forma curiosa los niños sanos en el primer año de vida, presentan valores hasta 10 veces superiores a los normales para niños mayores de dicha edad, sin existir una explicación clara al respecto (10). 


\section{Utilidad de los marcadores biológicos en la enfermedad inflamatoria intestinal}

Los denominados "Marcadores Biológicos de Actividad" o "Reactantes de Fase Aguda" se vienen empleando en el estudio y seguimiento de pacientes con enfermedad inflamatoria intestinal (EII) desde hace varias décadas, con diferentes objetivos entre los que hay que señalar con fines diagnósticos, para seguir la evolución del curso clínico, para realizar el diagnóstico diferencial con otros procesos intestinales y con la intención de monitorizar la eficacia del tratamiento empleado.

La llegada de los tratamientos biológicos en la EII, ha renovado el interés de la utilización de estos marcadores, especialmente la proteína $\mathrm{C}$ reactiva (PCR), por su utilidad para poder seleccionar pacientes que puedan responder a estos tratamientos.

De todos los reactantes de fase aguda, la PCR es el que más se ha estudiado y viene proporcionando excelentes resultados, ya que es un marcador objetivo de inflamación y se correlaciona bien con el grado de actividad, tanto en la enfermedad de Crohn (EC), como en la colitis ulcerosa (CU). Además presenta una vida media corta, por lo que se eleva precozmente al comienzo de la inflamación y disminuye paralelamente con su resolución. Su determinación es sencilla y rápida, ya que se realiza por un test de ELISA comercial siendo su determinación barata, sencilla y rápida, pudiendo realizarse incluso con carácter de urgencia. La presencia de niveles elevados de PCR se asocia con una mejor respuesta a los tratamientos biológicos y cuando están normales, por el contrario, predicen una mayor respuesta con el placebo.

Sin embargo, a pesar de las posibles ventajas de la determinación rutinaria de la PCR y otros marcadores, tienen también sus inconvenientes. Así por ejemplo, ha sido ampliamente demostrado que la PCR se relaciona mejor con la actividad de la enfermedad inflamatoria intestinal en pacientes con $\mathrm{EC}$, que en los que presentan una $\mathrm{CU}(11)$.

Otros marcadores de laboratorio entre los que se incluyen la determinación de la velocidad de sedimentación (V. de S), el recuento de leucocitos y de plaquetas, los niveles séricos de albúmina y de alfa-1-glicoproteína ácida (Orosomucoide), han sido estudiados en la EII y son por lo general menos útiles que la PCR. La V. de S. presenta varias desventajas entre las que se incluyen, el tener una vida media muy prolongada y que sus niveles dependen de muchos factores, tales como la edad, la presencia de anemia o policitemia, los niveles séricos de ciertas proteínas, incluso el tabaquismo y el tratamiento con diversos fármacos, por lo que su determinación actualmente no es comparable con la PCR en sensibilidad ni especificidad, para procesos inflamatorios $(12,13)$.

Los marcadores fecales parecen ser prometedores y más específicos para detectar la presencia de inflamación intestinal en pacientes con EII establecida. Se han observado resultados prometedores con el empleo de la calprotectina fecal (CPF), tanto en pacientes con EC como con CU. Datos recientes sugieren sin embargo, que la eficacia de la CPF, al contrario que la PCR, presenta una mayor sensibilidad diagnóstica en pacientes con CU, que con EC.

Analizados en conjunto, los marcadores biológicos de actividad inflamatoria son todos útiles y deben formar parte del manejo global del estudio y tratamiento de pacientes con EII. Sin embargo, hasta que no dispongamos de una mayor información, su utilización debe ser contemplada como una herramienta más de trabajo en estos pacientes con EII que se debe añadir a los datos de la observación clínica, exploración física, otras pruebas de laboratorio que junto con las pruebas de imagen, los hallazgos endoscópicos y biópsicos, siempre que sea posible y necesaria su utilización, ya que su empleo aislado nunca será suficiente ni llegará a sustituir o 
reemplazar un estudio completo y exhaustivo del paciente, empleando una gran mayoría de los diferentes medios diagnósticos que disponemos en la actualidad.

Algunos de estos nuevos métodos serológicos que se emplean en la EII parece que se asocian mejor con la enfermedad que presenta un curso complicado, como ha sido comunicado recientemente en un amplio estudio (14).

\section{Enfermedades orgánicas intestinales frente a funcionales}

Una de las dificultades diagnósticas frecuentes es la de separar entre casos leves o moderados con diversas causas de procesos inflamatorios intestinales, de los que no presentan una enfermedad orgánica.

Aunque los datos que proporciona la historia clínica junto con los de la exploración física y los hallazgos de las pruebas de laboratorio son a menudo orientadores, muchos pacientes necesitan ser explorados mediante endoscopia digestiva para confirmar o descartar dicho diagnóstico.

A este respecto la CPF es un marcador que refleja la presencia y el grado de inflamación intestinal. Su determinación ha demostrado una clara utilidad para ayudar a la realización del diagnóstico diferencial y en el seguimiento de pacientes, si bien su valor diagnóstico discriminativo no llega a alcanzar una potencia suficiente para poder separarlos en todos los casos.

Existen al menos 2 estudios prospectivos llevados a cabo en adultos que han estudiado estos aspectos. En dos de ellos los pacientes fueron enviados para diferenciar la EII con la patología funcional, principalmente el síndrome de colon irritable (SCI) $(15,16)$. También hemos recogido otros dos estudios realizados en niños con la misma orientación $(17,18)$.

Tibble y cols. estudiaron 220 pacientes adultos consecutivos para diferenciar EII de SCI. Utilizando la CPF con un nivel discriminativo de $10 \mathrm{mg} / \mathrm{l}$, la sensibilidad observada fue del $82 \%$ y su especificidad del $83 \%$ (15).

En otro estudio llevado a cabo por los mismos autores midieron la concentración fecal de calprotectina en 602 adultos, de los que 263 (44\%) fueron clasificados como enfermedad orgánica y los 339 restantes (56\%), como funcionales. La sensibilidad y especificidad de la CPF utilizando un valor umbral de $10 \mathrm{mg} / \mathrm{l}$, fue del $89 \mathrm{y}$ $79 \%$ respectivamente (16).

Los estudios realizados en niños con esta finalidad, han encontrado un mejor valor predictivo positivo y negativo que los observados en adultos, quizás debido a un menor tamaño muestral, por lo que sería deseable que sus resultados fuesen corroborados por otros autores y que incluyesen en sus trabajos un mayor número de pacientes $(17,18)$.

En el presente número de la Revista Española de Enfermedades Digestivas, Bonnín Tomás y cols. (19) determinan el valor de la CPF en una serie de 42 niños, comparando su utilidad en el diagnóstico y seguimiento de los mismos para la distinción entre patología orgánica y funcional y llegan a la conclusión de que se trata de un marcador sensible, pero no específico, que permite seleccionar pacientes con EII que requieren colonoscopia para su diagnóstico definitivo, disminuyendo o evitando su realización en pacientes con patología gastrointestinal funcional, por las incomodidades y dificultades que conlleva su realización en pacientes pediátricos.

En este mismo sentido se han realizado trabajos previos con idéntica finalidad y resultados muy similares (20). 
Se obtiene un mayor valor discriminativo en la separación entre enfermedades inflamatorias y funcionales cuando el valor umbral de la CPF se eleva a $100 \mu \mathrm{g} / \mathrm{g}$, como han confirmado recientemente algunos autores (21).

\section{Otras enfermedades intestinales y extra-intestinales}

En pacientes con colitis microscópica se han realizado diversos estudios que muestran por lo general valores elevados de $\mathrm{CPF}$, tanto en niños como en adultos, en un grado menor que en la EII (22).

En pacientes con enfermedad celiaca diagnosticada en cualquier edad de la vida, se observan valores elevados en aproximadamente el $50 \%$ de los casos, más frecuentemente cuando presentan enfermedad activa, que van disminuyendo progresivamente a partir del primer mes de instauración de una dieta sin gluten (23).

Sin embargo, un estudio publicado recientemente por Montalvo y cols. (24) en 22 pacientes celiacos adultos no tratados, no encontró diferencias significativas en los niveles de CPF con relación a los controles.

La CPF también se encuentra elevada en procesos extra-intestinales tales como la cirrosis hepática. Así en un estudio llevado a cabo sobre 53 pacientes los autores encuentran que los niveles de CPF estaban elevados en todos los pacientes, de forma progresiva en relación con el estadio funcional de Child-Pugh ( $p<0,001)$, con una tendencia a observar concentraciones más elevadas en pacientes con cirrosis alcohólica $(\mathrm{p}=0,1)$. Todo ello se puede relacionar con la presencia de un cierto grado de inflamación intestinal asociada, probablemente como consecuencia de la presencia de una traslocación bacteriana asociada (25).

Se encuentran valores elevados de CPF en pacientes con carcinoma colorrectal e igualmente en sujetos tratados recientemente con AINE aunque presenten una colonoscopia normal, como ha sido descrito recientemente por García Sánchez y cols. del Hospital Reina Sofía de Córdoba (26).

Conviene también recordar que en niños tratados con corticoides por una EII, los niveles de CPF disminuyen paulatinamente en relación con la mejoría clínica observada, permaneciendo elevados incluso varias semanas tras la finalización del tratamiento, lo que sugiere que existe una inflamación silente prolongada tras la mejoría clínica alcanzada (27).

Después de una resección completa del colon y recto con anastomosis íleo-anal, entre el $10-40 \%$ de los pacientes presentan una inflamación crónica del reservorio, tanto en adultos como en niños $(28,29)$.

Para seguir la evolución de la reservoritis se ha mostrado de utilidad la medición periódica de los niveles de $\mathrm{CPF}$, al igual que otros marcadores similares como la lactoferrina fecal $(30,31)$.

\section{Comentarios finales y conclusiones}

La medición de la CPF no está diseñada como un marcador de enfermedad orgánica intestinal, sino más bien es un parámetro de medición de inflamación intestinal neutrofílica.

Muchas enfermedades orgánicas intestinales, tales como la enfermedad celiaca, la diverticulosis del colon y el carcinoma de colon cuando se les estudian los niveles de este marcador, han producido resultados muy variables $(32,33)$. 
Por tanto, un resultado negativo de CPF no debería ser interpretado como equivalente de ausencia de patología orgánica intestinal (al menos en el adulto), sino más bien indicaría ausencia de inflamación intestinal por polinucleares neutrófilos.

Ello es de utilidad para diferenciar la EII del SCI. Sirve también si los síntomas que presenta un paciente con una enfermedad inflamatoria intestinal conocida, se deben a una reactivación de su enfermedad o a otros procesos, y ayuda también a valorar la respuesta al tratamiento de diversos fármacos en estos procesos.

L. Rodrigo

Servicio de Digestivo. Hospital Universitario Central de Asturias (HUCA). Oviedo

\section{Bibliografía}

1. Bjerke K, Halstensen TS, Jahnsen F, Pulford K, Brandtzaeg P. Distribution of macrophages and granulocytes expressing L1 protein (calprotectin) in human Peyer's patches compared with normal ileal lamina propria and mesenteric lymph nodes. Gut 1993; 34: 1357-63.

2. Røseth AG, Fagerhol MK, Aadland E, Schjønsby H. Assessment of the neutrophil dominating protein calprotectin in feces: a methodologic study. Scand J Gastroenterol 1992; 27: 793-8.

3. Bunn SK, Bisset WM, Main MJ, Golden BE. Fecal calprotectin as a measure of disease activity in childhood inflammatory bowel disease. J Pediatr Gastroenterol Nutr 2001; 32: 171-7.

4. Fagerberg UL, Lööf L, Merzoug RD, Hansson LO, Finkel Y. Fecal calprotectin levels in healthy children studied with an improved assay. J Pediatr Gastroenterol Nutr 2003; 37: 468-72.

5. Bjarnason I, Sherwood R. Fecal calprotectin: A significant step in the non-invasive assessment of intestinal inflammation. J Pediatr Gastroenterol Nutr 2001; 33: 11-3.

6. Bunn SK, Bisset WM, Main MJ, Gray ES, Olson S, Golden BE. Fecal calprotectin: validation as a non-invasive measure of bowel inflammation in childhood inflammatory bowel diseases. J Pediatr Gastroenterol Nutr 2001; 33: 14-22.

7. Summerton CB, Longlands MG, Wiener K, Shreeve DR. Faecal calprotectin: A marker of inflammation throughout the intestinal tract. Eur J Gastroenterol Hepatol 2002; 14: 841-5.

8. Tøn H, Brandsnes O, Dale S, Holtlund J, Skuibina E, Schjønsby H, et al. Improved assay for fecal calprotectin. Clin Chem Acta 2000; 292: 41-54.

9. Roseth AG, Schmidt PN, Fagerhol MK. Correlation between faecal excretion of indium-111-labeled granulocytes and calprotectin, a granulocyte marker protein in patients with inflammatory bowel disease. Scand $\mathbf{J}$ Gastroenterol 1999; 34: 50-4.

10. Campeotto F, Butel MJ, Kalach N, Derrieux S, Aubert-Jacquin C, Barbot L, et al. High fecal calprotectin concentrations in newborn infants. Arch Dis Child Fetal Neonatal Ed 2004; 89: F353-F354.

11. Vermeire S, Van Assche G, Rutgeerts P. Laboratory markers in IBD: Useful, magic, or unnecessary toys? Gut 2006; 55: 426-31.

12. Gisbert JP, González-Lama Y, Maté J. Utilidad de los marcadores biológicos en la enfermedad inflamatoria intestinal. Gastroenterol Hepatol 2007; 30: 117-29.

13. Sánchez Lombraña JL, Palacio MA, Carbajal C, Vivas S, Pérez R, Rodrigo L. Marcadores biológicos de actividad en la enfermedad inflamatoria intestinal crónica. Rev Esp Enferm Dig 1997; 89: 855-8.

14. Ferrante M, Henckaerts L, Joossens M, et al. New serological markers in inflammatory bowel disease are associated with complicated disease behaviour. Gut 2007; 56 : 1394-403.

15. Tibble J, Teahon K, Thjodleifsson B, et al. A simple method for assessing intestinal inflammation in Crohn's disease. Gut 2000; 47: 506-13.

16. Tibble JA, Sigthorsson G, Foster R, Forgacs I, Bjarnason I. Use of surrogate markers of inflammation and Rome criteria to distinguish organic from nonorganic intestinal disease. Gastroenterology 2002; 123: 450-60.

17. Carroccio A, Iacono G, Cottone M, et al. Diagnostic accuracy of fecal calprotectin assay in distinguishing organic causes of chronic diarrhea from irritable bowel syndrome: a prospective study in adults and children. Clin Chem 2003; 49 (6 Pt 1): 861-7.

18. Fagerberg UL, Lööf L, Myrdal U, Hansson LO, Finkel Y. Colorectal inflammation is well predicted by fecal calprotectin in children with gastrointestinal symptoms.J Pediatr Gastroenterol Nutr. 2005; 40: 450-5.

19. Bonnin Tomás A, Vila Vidal M, Rosell Camps A. Calprotectina fecal como marcador diferencial entre patología gastrointestinal orgánica y funcional. Rev Esp Enferm Dig 2007; 99: 689-93.

20. Bunn SK, Bisset WM, Main MJ, Gray ES, Olson S, Golden BE. Fecal calprotectin: Validation as a noninvasive measure of bowel inflammation in childhood inflammatory bowel disease. J Pediatr Gastroenterol Nutr 2001; 33: 14-22.

21. Von Roon AC, Karamountzos L, Purkayastha S, et al. Diagnostic precision of fecal calprotectin for inflammatory bowel disease and colorectal malignancy. Am J Gastroenterol 2007; 102: 803-13. 
22. Limburg PJ, Ahlquist DA, Sandborn WJ, et al. Fecal calprotectin levels predict colorectal inflammation among patients with chronic diarrhea referred for colonoscopy. Am J Gastroenterol 2000; 95: 2831-7.

23. Berni Canani R, Rapacciuolo L, Romano MT, et al. Diagnostic value of faecal calprotectin in paediatric gastroenterology clinical practice. Dig Liver Dis 2004; 36: 467-70.

24. Montalto M, Santoro L, Curigliano V, et al. Faecal calprotectin concentrations in untreated coeliac patients. Scand J Gastroenterol 2007; 42: 957-61.

25. Yagmur E, Schnyder B, Scholten D, et al. Elevated concentrations of fecal calprotectin in patients with liver cirrhosis. Dtsch Med Wochenschr 2006; 131: 1930-4.

26. García Sánchez M del V, González R, Iglesias Flores E, et al. Valor diagnóstico de la calprotectina fecal para predecir una colonoscopia alterada. Med Clin (Barc) 2006; 127: 41-6.

27. Kolho KL, Raivio T, Lindahl H, Savilahti E. Fecal calprotectin remains high during glucocorticoid therapy in children with inflammatory bowel disease. Scand J Gastroenterol 2006; 41: 720-5.

28. Lohmuller JL, Pemberton JH, Dozois RR, Ilstrup D, Van Heerden J. Pouchitis and extraintestinal manifestations of inflammatory bowel disease after ileal pouch-anal anastomosis. Ann Surg 1990; 211: 622-7.

29. Stavlo PL, Libsch KD, Rodeberg DA, Moir CR. Pediatric ileal pouch-anal anastomosis: functional outcomes and quality of life. J Pediatr Surg 2003; 38: 935-9.

30. Thomas P, Rihani H, Røseth A, et al. Assessment of ileal pouch inflammation by single-stool calprotectin assay. Dis Colon Rectum 2000; 43: 214-20.

31. Parsi MA, Shen B, Achkar JP, et al. Fecal lactoferrin for diagnosis of symptomatic patients with ileal pouchanal anastomosis. Gastroenterol 2004; 126: 1280-6.

32. Hoff G, Grotmol T, Thiis-Evensen E, Bretthauer M, Gondal G, Vatn MH. Testing for faecal calprotectin (Phi$\mathrm{Cal}$ ) in the Norwegian Colorectal Cancer Prevention trial on flexible sigmoidoscopy screening: Comparison with an immunochemical test for occult blood (FlexSure OBT). Gut 2004; 53: 1329-33.

33. Limburg PJ, Devens ME, Harrington JJ, Diehl NN, Mahoney DW, Ahlquist DA. Prospective evaluation of fecal calprotectin as a screening biomarker for colorectal neoplasia. Am J Gastroenterol 2003; 98: 2299-305. 